\title{
Pericardial Tamponade Caused by Rotational Atherectomy Ablation Grinding Through a Barrier Stent Protruding From the Diagonal Artery: A Case Report
}

\author{
Ming Lian Gong ${ }^{\mathrm{a}, \mathrm{b}}$, Yi Mao ${ }^{\mathrm{c}, \mathrm{d}}$, Jing Hua Liu ${ }^{\mathrm{b}, \mathrm{d}}$
}

\begin{abstract}
Rotational atherectomy (RA) has been described in case reports to be effective in ablating under-expanded stents. We present a case of calcified bifurcation lesion percutaneous coronary intervention (PCI), in which the branch stent protruding into main branch blocked the balloon getting through, and the following RA led to pericardial tamponade. The first stent was deployed from the proximal of left anterior descending artery (LAD) to the proximal of diagonal for an 80-yearold man who suffered from non-ST-elevation myocardial infarction to make a Culotte dual stent PCI. The operator failed to make balloon get through the struts protruding into LAD lumen anyway. After RA drill through the LAD lumen, it showed an excellent angiographic outcome. Stent deployment in LAD was successful, but pericardial tamponade occurred in a short time. After pericardiocentesis was performed under fluoroscopic guidance, the patient was uneventful. After 1 year, the follow-up coronary computed tomography (CT) angiography showed no in-stent restenosis, stent fracture or disrupted struts protruding outside of the vessel's outline of LAD and the first diagonal (D1). This case shows stents' RA could be dangerous in grinding across the stents' lateral hole in an unsymmetrical lumen. Although RA could be a useful remedy in the situation of under-expansion of implanted stents, the debulking should be performed for longitudinal stent ablation and cautiously performed for bifurcation lesion in which the protruding stents from side branch were unsym-
\end{abstract}

Manuscript submitted February 7, 2021, accepted February 23, 2021

Published online March 24, 2021

aDepartment of Cardiology, Dalian The Fifth People's Hospital, Dalian, China bepartment of Cardiology, Beijing Anzhen Hospital, Capital Medical University, Beijing Institute of Heart, Lung and Blood Vessel Diseases, Beijing, China

'Department of Cardiology, Fuwai Hospital, National Center for Cardiovascular Disease, Chinese Academy of Medical Science and Peking Union Medical College, Beijing, China

${ }^{\mathrm{d}}$ Corresponding Author: Yi Mao, Department of Cardiology, Fuwai Hospital, Chinese Academy of Medical Science and Peking Union Medical College, National Center for Cardiovascular Disease, No.167 Beilishi Road, Xicheng District, Beijing 100037, China. Email: 13693305832@139.com; Jing Hua Liu, Department of Cardiology, Beijing Anzhen Hospital, Capital Medical University, Beijing Institute of Heart, Lung and Blood Vessel Diseases, No. 2 Chaoyang Road, Chaoyang District, Beijing 100029, China.

Email: liujinghua@vip.sina.com

doi: https://doi.org/10.14740/jmc3678 metrically blocking the path.

Keywords: Rotational atherectomy; Pericardial tamponade; Stent under-expansion; Coronary angiography

\section{Introduction}

Rotational atherectomy (RA) has been described in case reports to be effective in ablating under-expanded stents $[1,2]$. However, there are no reports RA were used in grinding off the barrier struts from the branch. We present a case of calcified bifurcation lesion percutaneous coronary intervention (PCI), in which the branch stent protruding into main branch blocked the balloon getting through, and the following RA make a complication of pericardial tamponade.

\section{Case Report}

An 80-year-old man with non-ST-elevation myocardial infarction was presented to our hospital, with a history of hypertension and dyslipidemia. Coronary angiography revealed diffuse calcification from proximal to middle segment of the left anterior descending artery (LAD) with about $80 \%$ stenosis and $80 \%$ stenosis in the proximal of diagonal. Considering the LAD true bifurcation lesion and similar vessel diameter of LAD and the first diagonal (D1), we planned to utilize culotte technique. A drug-eluting stent (DES, $2.5 \times 15 \mathrm{~mm}$ ) was implanted in the D1 with short main branch protrusion, then no balloons could pass through the first stent's side hole (Fig. 1). Based on current RA recommendations, the burr-to-artery ratio was 0.5 [3]. RA with $1.5 \mathrm{~mm}$ burr was attempted, and the ablation was initiated at the proximal edge of the stent. Following eight runs of RA with a speed of $160,000 \mathrm{rpm}$ and post-dilatation of a $3.0 \times 12 \mathrm{~mm}$ noncompliant (NC) balloon at $20 \mathrm{~atm}$, a full expansion with a good angiographic result was achieved. Another DES $(3.0 \times 30 \mathrm{~mm})$ was implanted in the LAD with full expansion and followed with the dilation of $\mathrm{NC}$ balloon $(3.0 \times 12 \mathrm{~mm})$ at $26 \mathrm{~atm}$ (Fig. 2). Final angiography showed a satisfactory result with no stent malposition or residual stenosis. Half an hour after PCI, the patient felt shortness of breath with low blood pressure. Ultrasound cardiogram showed 

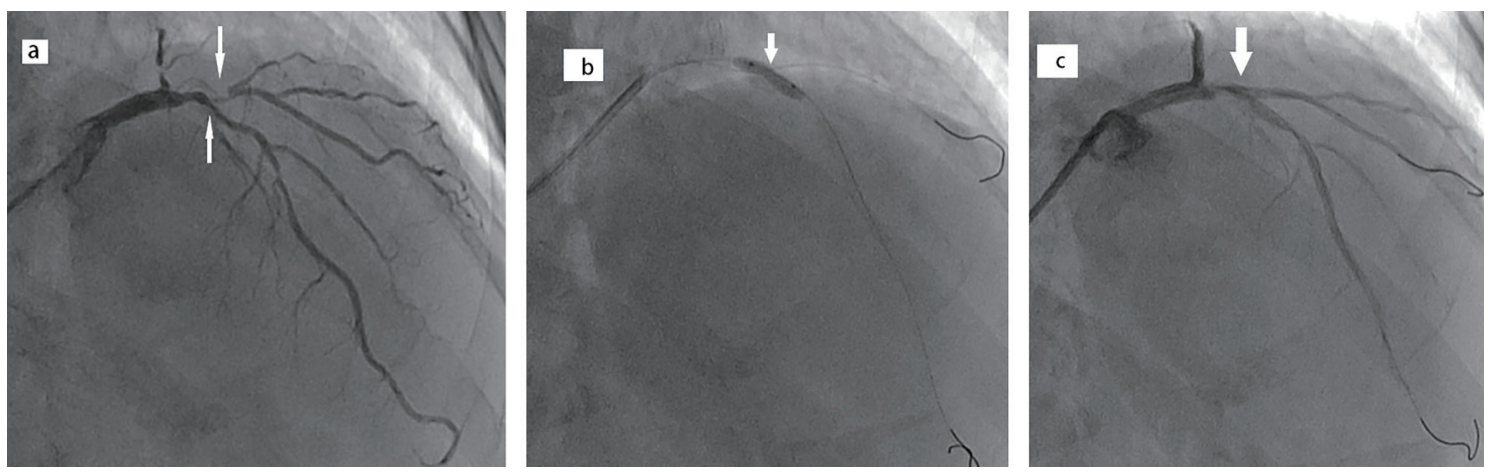

Figure 1. Angiography and under-expanded balloon and stent under-expansion. (a) Baseline coronary angiogram of left anterior descending artery (LAD) and first diagonal (D1). (b) Balloon expansion at high pressure failed to expand the lesion. (c) The first stent was deployed from the proximal of LAD to the proximal of diagonal with under-expansion.

pericardium effusion and angiography showed no obvious coronary perforation. After pericardiocentesis was performed under fluoroscopic guidance, the patient was uneventful. The follow-up ultrasound cardiogram showed no more pericardium effusion with normal ventricular wall motion. The patient received the standard medical therapy with doctor's prescription of aspirin, clopidogrel, angiotensin-converting enzyme inhibitor (ACEI), beta-blocker, statin drugs and outpatient followup once a month after discharge. After 1 year, the follow-up coronary computed tomography (CT) angiography showed no in-stent restenosis, stent fracture or disrupted struts protruding outside of the vessel's outline of LAD and D1.

\section{Discussion}

There are limited options for stent under-expansion due to rig-

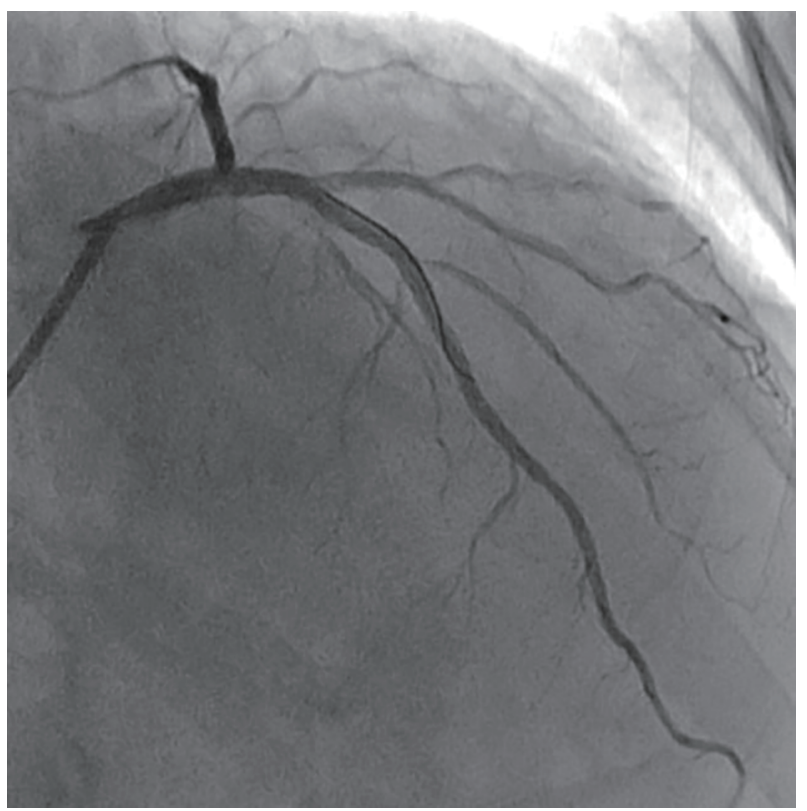

Figure 2. Final result. The final angiogram showed fully expanded left anterior descending artery (LAD) and first diagonal (D1). id calcified lesions. RA has been described in case reports to be practical and effective in ablating under-expanded stents $[4,5]$. Compared to previously reported cases, our case illustrates the risk of pericardial tamponade after ablation of the barrier stent struts in the calcified bifurcation lesion with RA. A rotational speed of 140,000 to $150,000 \mathrm{rpm}$ was the ideal speed that led to fewer complications [3]. In our case, RA debulked both the struts protruding from side branch and calcified plaques. Due to the stiffer metallic scaffold, higher speed RA was needed to avoid burr entrapment according to our experience. When RA is performed, lesion specific predictors of perforation include eccentricity tortuosity, length $>10 \mathrm{~mm}$ and location in the right coronary artery or left circumflex artery [3]. In our case, ablating the struts protruding from side branch was not same as longitudinal stent ablation in a symmetrical lumen. Due to the incomplete Stent ablation in an unsymmetrical lumen, the remaining stent scaffold outward protruding was apt to injure the vessel wall and cause invisible impairment which resulted in pericardial tamponade. It was also a common phenomenon in the coronary chronic total occlusion PCI, non-contrast staining but tamponade happened. Although RA could be a useful remedy in the situation of under-expansion of implanted stents, the debulking should be performed for longitudinal stent ablation and cautiously performed for bifurcation lesion in which the protruding stents from side branch were unsymmetrically blocking the path.

\section{Acknowledgments}

None to declare.

\section{Financial Disclosure}

None to declare.

\section{Conflict of Interest}

None to declare. 


\section{Informed Consent}

Informed consent was obtained from the patient for publication of this case report and accompanying images.

\section{Author Contributions}

Manuscript conception and design: YM. Acquisition of data: MLG. Analysis and interpretation of data (e.g. statistical analysis computational analysis): MLG. Writing, review and/or revision of the manuscript: MLG. Manuscript supervision: JHL, YM.

\section{Data Availability}

Any inquiries regarding supporting data availability of this study should be directed to the corresponding author.

\section{Abbreviations}

DES: drug-eluting stent; LAD: left anterior descending artery;
D: diagonal; NC: noncompliant; PCI: percutaneous coronary intervention; RA: rotational atherectomy

\section{References}

1. Medina A, de Lezo JS, Melian F, Hernandez E, Pan M, Romero M. Successful stent ablation with rotational atherectomy. Catheter Cardiovasc Interv. 2003;60(4):501504.

2. Koide M, Inoue K, Matsuo A, Fujita H. Optical coherence tomography findings after longitudinal ablation for an underexpanded stent in a heavily calcified lesion: a case report. BMC Cardiovasc Disord. 2016;16(1):241.

3. Sharma SK, Tomey MI, Teirstein PS, Kini AS, Reitman AB, Lee AC, Genereux P, et al. North American Expert review of rotational atherectomy. Circ Cardiovasc Interv. 2019;12(5): e007448.

4. Hernandez J, Galeote G, Moreno R. Rotational atherectomy: if you do not do it before, you can do it after stenting. J Invasive Cardiol. 2014;26(9):E122-123.

5. Si D, Liu G, Tong Y, He Y. Rotational atherectomy ablation for an unexpandable stent under the guide of IVUS: A case report. Medicine (Baltimore). 2018;97(7):e9978. 